\title{
T wave positivity in lead aVR is associated with mortality after transcatheter aortic valve implantation
}

Yurdaer Dönmez, Örsan Deniz Urgun, İbrahim Halil Kurt

Department of Cardiology, University of Health Sciences - Adana Health Practices and Research Centre, Adana, Turkey

Submitted: 23 January 2019

Accepted: 31 March 2019

Arch Med Sci Atheroscler Dis 2019; 4: e55-e62

DOI: https://doi.org/10.5114/amsad.2019.84449

Copyright $\odot 2019$ Termedia \& Banach

\section{Abstract}

Introduction: Transcatheter aortic valve implantation (TAVI) is an alternative to surgical aortic valve replacement (SAVR) in patients with increased comorbidity. Lead aVR in surface ECG has valuable information about prediction of mortality in many cardiovascular diseases. Our aim was to determine the relationship between ischemic changes in lead aVR and mortality in TAVI patients.

Material and methods: We retrospectively examined 86 patients with TAVI. The ST segment deviation in lead aVR (STaVR) and T wave polarity (TPaVR) on surface ECG were measured. The absolute values of TPaVR and STaVR were calculated. A ratio (TP/STaVR or vice versa) was obtained from the division of the larger absolute value by the smaller one.

Results: The patients were divided into two groups as living and deceased. The living group had 68 patients, and the deceased group had 18 patients. The number of positive TPaVR patients after TAVI, TPaVR after TAVI, and TP/ STaVR ratio after TAVI were significantly higher in the deceased group. The deceased group had a significantly shorter aortic annulus-LMCA distance. Presence of positive TPaVR (OR $=8.765,95 \% \mathrm{Cl}: 1.088-70.618, p=0.041)$, aortic annulus-LMCA distance (for each $1 \mathrm{~mm}$ increase, $\mathrm{OR}=0.306$, 95\% Cl: $0.158-0.595, p<0.001$ ) and TP/STaVR ratio (for each 0.1 increase, $\mathrm{OR}=1.966,95 \% \mathrm{Cl}: 1.276-3.024, p=0.002$ ) were determined as independent predictors for mortality.

Conclusions: Ischemic changes in lead aVR may provide valuable information about mortality after TAVI.

Key words: electrocardiogram, lead aVR, mortality, transcatheter aortic valve implantation.

\section{Introduction}

In developed countries, the number of patients with heart valve diseases increases as the population gets older [1]. Aortic valve replacement (AVR) has favorable effects on mortality and morbidity in patients with severe symptomatic aortic stenosis. Percutaneous transcatheter aortic valve implantation (TAVI) began to be used in the early 2000s. At first, it was advised to perform it in high-volume centers and selected patients [2]. Today, it is recommended as a class 1 indication in current guidelines [3]. Transcatheter aortic valve implantation (TAVI) is an alternative to surgical aortic valve replacement (SAVR) in patients with a Society of Thoracic Surgeons Probability of Mortality (STS-PROM) score $\geq 8$ [4].

\author{
Corresponding author: \\ Yurdaer Dönmez \\ Department \\ of Cardiology \\ Adana Health Practices \\ and Research Center \\ Health Sciences \\ University \\ 01000 Adana, Turkey \\ Phone: +90 5332567896 \\ E-mail:yurdaerd@gmail.com
}


With the advances in TAVI methods and devices, mortality rates were reported as $1.1-4.2 \%$ in multicenter randomized trials [5-7]. In patients selected by the heart team, mortality rate diminishes with the appropriate access point and technique, suitable valve type and size, experienced operators, and meticulous vascular closure methods.

Despite modern imaging techniques and diagnostic methods, 12-lead surface electrocardiogram (ECG) still presents beneficial information regarding upcoming cardiac events. There are reports of negative effects on mortality of newly developed left bundle branch block (LBBB), complete atrioventricular block and QRS complex enlargement in patients with TAVI [8-10].

Lead aVR in surface ECG is a neglected lead in routine daily practice. However, it provides valuable information about the prediction of mortality in many cardiovascular diseases [11]. It is reported that ST segment and T wave changes in lead aVR have negative effects on mortality in patients with ST-elevation myocardial infarction, non-ST elevation myocardial infarction and heart failure [12-14]. Changes in lead aVR may provide information about global ischemia in the left ventricle (LV) [15]. After TAVI, left ventricular functions may be affected due to the proximity of the main coronary artery to the aortic valve. Therefore, ischemic changes may appear in the lead aVR. There is little information about the relation between lead aVR changes and mortality in patients with TAVI. We aimed to determine the relationship between lead aVR changes in pre- and post-procedural surface ECGs and mortality in TAVI patients.

\section{Material and methods}

\section{Patient population}

Cukurova University Medical School Ethical Committee approved our study. We retrospectively examined 103 patients who underwent a TAVI procedure because of severe aortic stenosis (aortic valve area (AVA) $\leq 1 \mathrm{~cm}^{2}$ or index AVA $\leq 0.6 \mathrm{~cm}^{2} / B S A$ or mean gradient $\geq 40 \mathrm{~mm} \mathrm{Hg}$ or aortic velocity $\geq 4 \mathrm{~m} / \mathrm{s}$ during the period 2015-2018. Some of the patients were excluded: 6 with atrial fibrillation (AF), 2 immediately deceased after the procedure, 2 non-cardiac deaths (possible stroke), 5 permanent pacemaker implantations due to complete atrioventricular block, and 2 who had insufficient information. We enrolled 86 patients and recorded their demographic variables. Information about patients' current medical status was obtained from the hospital follow-ups. If the patient was not able to come to hospital, then subjects' vital status was learned via phone.

\section{Evaluation of laboratory findings}

Complete blood count, glucose, calcium, phosphorus, renal functions, glomerular filtration rate, uric acid, total protein, albumin, high-sensitivity troponin $\mathrm{T}$ (hs-TnT), N-terminal pro-brain natriuretic peptide (NT-proBNP), CK-MB, high-sensitivity C reactive protein (hs-CRP) and thyroid functions were recorded from routinely taken blood samples.

\section{Electrocardiographic evaluation}

Pre- and post-procedural (after 24 h) 12-lead surface ECGs of all patients were recorded ( $\mathrm{Ni}$ hon Kohden Cardiofax V model ECG-1550K device). Electrocardiograms had $25 \mathrm{~mm} / \mathrm{s}$ speed and $1 \mathrm{mV} / 10 \mathrm{~mm}$ standard calibration. These ECGs were assessed by two independent cardiologists (Figures $1 \mathrm{~A}, \mathrm{~B}$ ). QRS duration and axis, $\mathrm{P}$ wave duration, PR interval, QT and QTc durations, and existence of LBBB were recorded. Negative or positive numeric data were recorded according to the ST segment's location being under or above the isoelectric line in lead aVR (STaVR). T wave polarity (TPaVR) was determined according to the peak of the T wave and the vertical distance to the isoelectric line. If the $T$ wave was below the isoelectric line then negative values were recorded. Positive values were recorded when the $T$ wave was above the isoelectric line. In the case of a biphasic $T$ wave, total vector magnitude was calculated and noted. The absolute values of TPaVR and STaVR were calculated. Then, the previously described ratio was obtained from the division of the larger absolute value by the smaller one (TP/STaVR ratio or vice versa: (larger value)/(smaller value)) [14].

\section{Echocardiographic evaluation}

Transthoracic echocardiography (TTE) and transesophageal echocardiography (TOE) were applied before the procedure (Epiq 7, Philips Healthcare, DA Best, Netherlands). Only transthoracic echocardiography (TTE) was performed after the procedure. Ejection fraction (EF), left atrium diameter (LaD), aortic valve gradient, aortic valve area, and systolic pulmonary artery pressure were measured with TTE. Diameters of ascending aorta sinotubular junction, sinus Valsalva diameter, and distance between the aortic annulus and the left main coronary artery (LMCA) were measured with TOE before TAVI. Prosthetic valve gradient and paravalvular leak were evaluated by post-procedural TTE.

\section{Angiographic evaluation}

Coronary angiograms were performed with the Judkins technique. Two independent cardiologists evaluated the angiograms. All the critical lesions were recorded. Anatomical SYNTAX scores were calculated. All the arteries with $\geq 1.5 \mathrm{~mm}$ diam- 


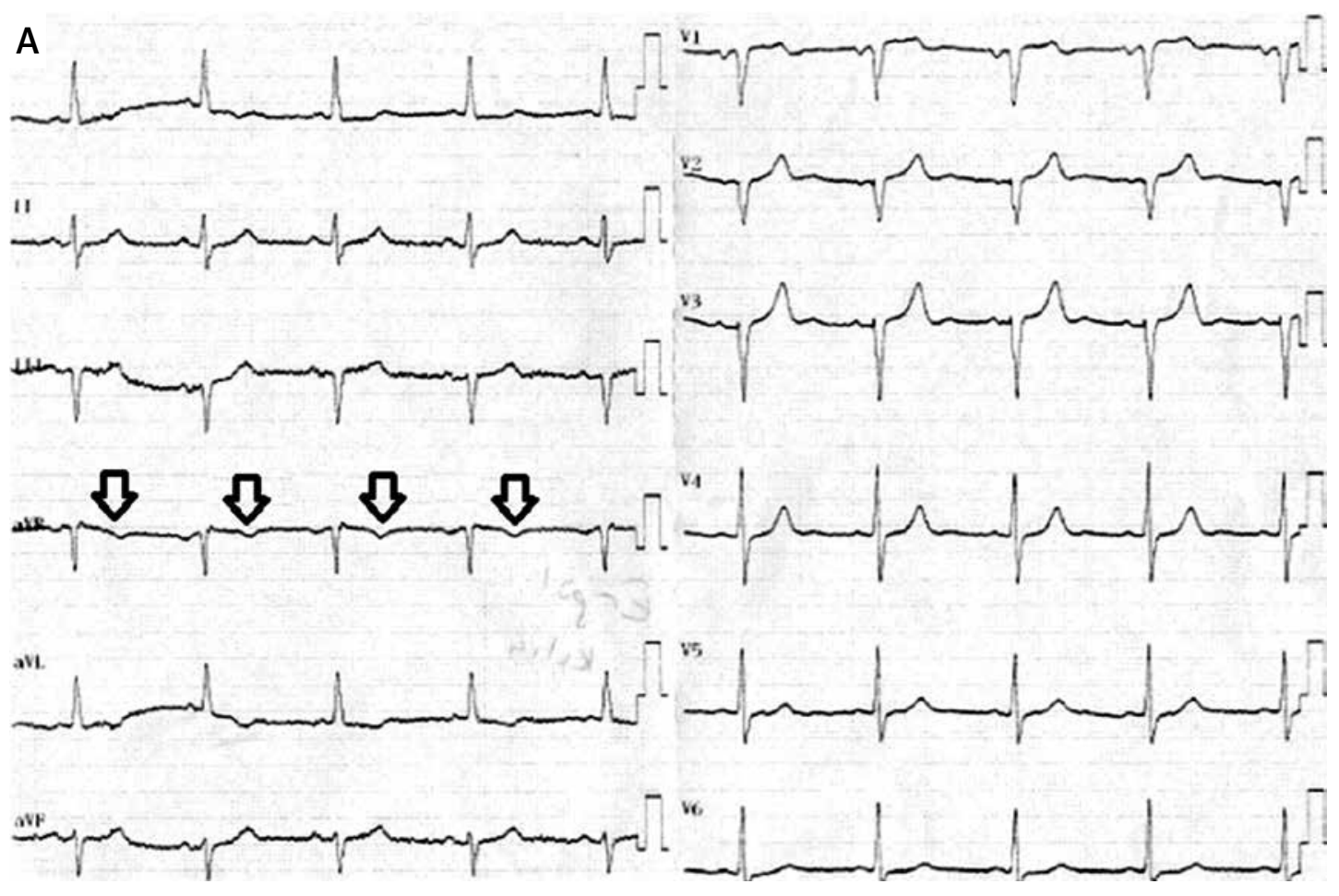

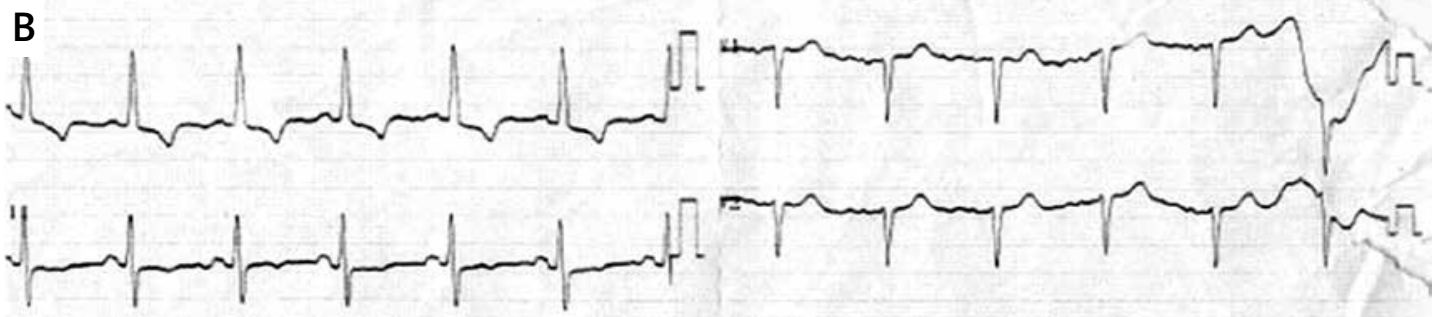<smiles>CCCCCC(C)CCC(C)CC(C)CCC(C)CCC(C)CC(C)CC(C)CC(C)CCC(C)CCCCC</smiles>

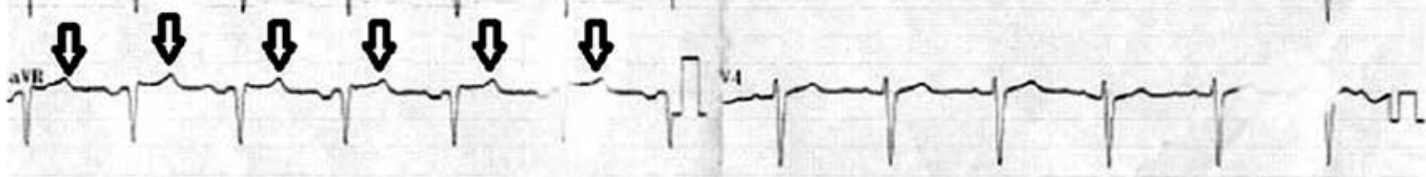

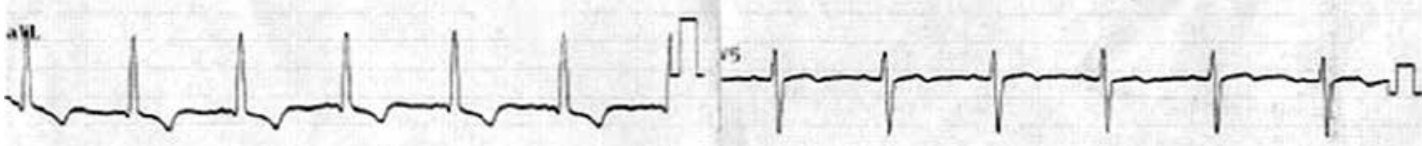

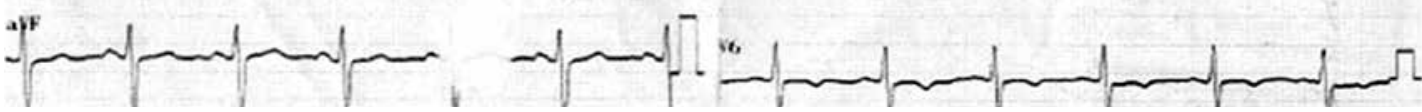

Figure 1. A - Demonstration of negative T wave on surface ECG before transcatheter aortic valve implantation,

B - demonstration of positive T wave on surface ECG after transcatheter aortic valve implantation

eter and over $50 \%$ stenosis were included in this calculation.

\section{Transcatheter aortic valve implantation procedure}

Symptomatic severe aortic stenosis patients with a high STS-PROM score (STS $\geq 8$ ), porcelain aorta, previous cardiac surgery history or previous chest radiotherapy history were evaluated for the TAVI procedure. The final decision was given by the heart team. The team consisted of an invasive cardiologist, heart surgeon, and anesthesiologist. Femoral, iliac and aortic anatomies were evaluated with the standard invasive angiogram or computed tomography angiography. Femoral 
access was used in all patients. The aortic valve was crossed by the Amplatzer Left (AL) 1-2 catheter and a $260 \mathrm{~cm}$ stiff guidewire was placed into the left ventricle. After the wire was crossed the valve, the catheter was advanced over the wire in right anterior oblique view and positioned in the middle of the left ventricle. An extra-stiff Amplatz 0.035 inch, $270 \mathrm{~cm}$ long guidewire (Cook, Bjaeverskov, Denmark) was used to perform all catheter exchanges and to assist stabilizing the valvuloplasty balloon $(20-25 \times 45 \mathrm{~mm}$ Z-Med II balloon catheter, NuMed, Hopkinton NY) during inflation, deflation, and withdrawal. The contrast was diluted (15\% contrast and $85 \%$ saline) to reduce viscosity for facilitation of the inflation and deflation cycles.

A temporary 6-Fr bipolar pacing lead was placed in the right ventricular posterior wall and connected to a pulse generator capable of pacing at up to $180-220$ beats/min. The pacer was set to on standby mode at 80 beats/min as a backup in case of a bradycardia or asystole in response to balloon inflation. Then, temporary pacing was applied with 140-180 bpm/ min rates, and an appropriate sized prosthetic aortic valve was implanted into the aortic annulus. A self-expandable prosthetic valve was used in all patients. If there was aortic regurgitation, post-dilatation was applied. Paravalvular leak was also checked in the fluoroscopic views. Activated clotting time was maintained around 250-300 s throughout the procedure by heparin infusion. A percutaneous closure device was used at the end of the procedure (Perclose Proglide Suture-mediated Closure System, Abbott, Abbott Park, IL, USA). Patients were followed up for 24 hours in the coronary intensive care unit.

\section{Statistical analysis}

Variables were divided into categorical and continuous groups. Categorical variables were expressed as numbers and percentages. The $\chi^{2}$ test was used to analyze categorical variables. Continuous variables were shown as mean and standard deviation. The Kolmogorov-Smirnov test was used to determine whether continuous variables had a normal distribution or not. Normally distributed variables were analyzed by the independent samples $t$-test. Non-normally distributed variables were analyzed by the Mann-Whitney $U$ test. Independent predictors for mortality were determined by the binomial logistic regression analysis using $p<0.05$ as the criterion. The program SPSS for Windows 20.0 (SPSS, Armonk, New York, USA) was used for statistical analysis. A $p<0.05$ was considered to be statistically significant.

\section{Results}

The patients were divided into two groups as living and deceased. The living group had 68 patients (mean age: $75.4 \pm 8.4$ years, mean follow-up period: $15.0 \pm 11.4$ months) and the deceased group had 18 patients (mean age: $73.7 \pm 7.0$ years, mean follow-up period: $14.7 \pm 8.7$ months). Three patients died within the first week of the procedure, and all of them had a positive $T$ wave in lead aVR. The other deceased patients were followed up for at least 30 days. There were no significant differences in demographic variables between the two groups (Table I). In the comparison of laboratory findings, the living group had a significantly higher albumin level $(p=0.012)$, and the deceased group had a significant higher calcium level ( $p=0.029$ ) (Table I). When electrocardiographic data were compared, the number of positive TPaVR patients after TAVI $(p=0.009)$, TPaVR after TAVI $(p=0.039)$ and TP/STaVR ratio after TAVI $(p=0.002)$ were significantly higher in the deceased group (Table II). The deceased group had a significantly shorter aortic annulus-LMCA distance $(p<0.001)$, and all other echocardiographic measurements were similar (Table III). Coronary angiographic findings were similar in both groups (Table IV). In the binomial logistic regression analysis, presence of positive TPaVR $(\mathrm{OR}=8.765,95 \% \mathrm{Cl}: 1.088-70.618, p=0.041)$, aortic annulus-LMCA distance (for each $1 \mathrm{~mm}$ increase, $\mathrm{OR}=0.306,95 \% \mathrm{Cl}$ : 0.158-0.595, $p<$ 0.001 ) and TP/STaVR ratio (for each 0.1 increase, $\mathrm{OR}=1.966,95 \% \mathrm{Cl}: 1.276-3.024, p=0.002)$ were determined as independent predictors for mortality (Table V).

\section{Discussion}

Our study was the first to investigate the lead aVR findings in TAVI patients and had some important findings. First, the presence of positive TPaVR after TAVI was closely associated with mortality. Second, TP/STaVR ratio of the ST segment and $T$ wave changes in lead aVR of post-procedural ECGs were closely related to mortality. Third, the increase in aortic annulus-LMCA distance was related to decreased mortality.-

Lead aVR reflects the left ventricle apex vectorially. It is known as a highly sensitive lead for ischemia in this area. It also gives information about global ischemia in the left ventricle $[11,15]$. The TAVI applicable patients have high STS score and more comorbidities. Balloon valvuloplasty applies a mechanical pressure and smashes calcifications in the aortic valve. This calcified microparticles spread mainly to the coronary arteries and arterial vascular tree. Balloon predilatation of the aortic valve generates a temporary pressure over the left main coronary artery, and this may lead to deteri- 
oration in the oxygen supply. Fast pacing-induced hypotension can also increase oxygen supply deficit related myocardium damage. To us, the most dramatic phase of the procedure is implantation of the valve. It also has the most catastrophic damage potential. If the valve is placed over or near to the LMCA ostium, then the coronary flow of the left ventricle can be severely affected. It was reported that the TAVI originated myocardial damage closely associated with mortality [16-18].

In our study, the deceased group had a significantly shorter aortic annulus-sinus Valsalva distance than the living group. Both groups had similar and low SYNTAX scores, but due to this shorter distance, the prosthetic valve could have applied a chronic pressure on the LMCA. Such probable pressure over the LMCA may play a critical role in the subclinical ischemia of the left ventricle and may cause increased mortality. Deceased patients could have died due to ischemic or arrhythmic results of this possible pressure over the LMCA ostium. We found that TPaVR and TP/STaVR ratio in lead aVR were increased significantly in the deceased group. Stundl et al. reported that myocardial damage was dependent on valve type, but there was no association with mortality [19]. Post-procedural hs-TnT levels were higher in the deceased group, but there was no significant difference. Maybe we would be able to see a difference in the hs-TnT levels if we had continued to make serial measurements.

It is necessary to choose the right patient group for lower mortality and morbidity in the TAVI procedure. Also, proper medications should be given very carefully to these patients in order to reduce complications. Antithrombotics and anticoagulants may have very deteriorative effects in this fragile patient group [20]. It has been mentioned that the STS-PROM scoring system was developed for the SAVR and its reliability was low for the TAVI patient group. Acute kidney damage, periprocedural myocardial infarction, low EF, and increased

Table I. Comparison of patients' demographic and laboratory findings

\begin{tabular}{|c|c|c|c|}
\hline Parameter & Living $(n=68)$ & Deceased $(n=18)$ & $P$-value \\
\hline Age [years] & $75.4 \pm 8.4$ & $73.7 \pm 7.0$ & 0.434 \\
\hline Male gender, $n(\%)$ & $29(42.6)$ & $5(27.8)$ & 0.251 \\
\hline Body mass index $\left[\mathrm{kg} / \mathrm{m}^{2}\right]$ & $27.7 \pm 5.1$ & $28.6 \pm 1.8$ & 0.732 \\
\hline Smoking, $n(\%)$ & $18(26.5)$ & $6(33.3)$ & 0.564 \\
\hline Diabetes mellitus, $n(\%)$ & $20(29.4)$ & $6(33.3)$ & 0.747 \\
\hline Hypertension, $n$ (\%) & $50(73.5)$ & $13(72.2)$ & 0.911 \\
\hline Hyperlipidemia, n (\%) & $18(26.5)$ & $4(22.2)$ & 0.713 \\
\hline Stroke, $n(\%)$ & $6(8.8)$ & $0(0)$ & 0.191 \\
\hline CAD, $n(\%)$ & $28(41.2)$ & $10(55.6)$ & 0.275 \\
\hline COPD, $n(\%)$ & $11(16.2)$ & $6(33.3)$ & 0.104 \\
\hline STS-PROM score, $n(\%)$ & $10.7 \pm 1.8$ & $10.8 \pm 2.0$ & 0.802 \\
\hline Glucose [mg/dl] & $132.9 \pm 50.8$ & $141.1 \pm 31.5$ & 0.526 \\
\hline Hemoglobin [mg/dl] & $11.7 \pm 1.7$ & $10.9 \pm 0.7$ & 0.054 \\
\hline $\mathrm{BUN}[\mathrm{mg} / \mathrm{dl}]$ & $50.2 \pm 16.1$ & $53.4 \pm 13.8$ & 0.438 \\
\hline Creatinine [mg/dl] & $1.1 \pm 0.6$ & $1.3 \pm 0.9$ & 0.242 \\
\hline Glomerular filtration rate $\left[\mathrm{ml} / \mathrm{min} / \mathrm{m}^{2}\right]$ & $72.6 \pm 18.8$ & $63.7 \pm 18.6$ & 0.083 \\
\hline Total protein [g/dl] & $6.2 \pm 0.6$ & $6.1 \pm 0.4$ & 0.379 \\
\hline Albumin $[\mathrm{g} / \mathrm{dl}]$ & $3.5 \pm 0.4$ & $3.2 \pm 0.5$ & 0.012 \\
\hline Calcium [mg/dl] & $8.7 \pm 0.7$ & $9.1 \pm 0.5$ & 0.029 \\
\hline $\mathrm{Hs}-\mathrm{CRP}[\mathrm{mg} / \mathrm{l}]$ & $1.5 \pm 2.1$ & $1.7 \pm 0.6$ & 0.248 \\
\hline NT-proBNP [pg/ml] & $2531 \pm 1580$ & $2884 \pm 1171$ & 0.581 \\
\hline High sensitive troponin $\mathrm{T}[\mathrm{pg} / \mathrm{l}]$ & $0.09 \pm 0.15$ & $0.14 \pm 0.19$ & 0.287 \\
\hline
\end{tabular}

$B U N$ - blood urea nitrogen, CAD - coronary artery disease, COPD - chronic obstructive pulmonary disease, hs-CRP - high-sensitivity C-reactive protein, NT-proBNP - N-terminal brain natriuretic peptide, STS-PROM - Society of Thoracic Surgeons Probability of Mortality. 
Yurdaer Dönmez, Örsan Deniz Urgun, İbrahim Halil Kurt

Table II. Comparison of patients' electrocardiographic findings

\begin{tabular}{|c|c|c|c|}
\hline Parameter & Living $(n=68)$ & Deceased $(n=18)$ & $P$-value \\
\hline QRS before TAVI [msn] & $100.7 \pm 20.6$ & $91.6 \pm 17.2$ & 0.13 \\
\hline QRS after TAVI [msn] & $120.1 \pm 23.9$ & $105.4 \pm 23.4$ & 0.101 \\
\hline$P$ duration before TAVI [ms] & $111.4 \pm 25.4$ & $108.3 \pm 19.7$ & 0.624 \\
\hline P duration after TAVI [ms] & $105.3 \pm 9.3$ & $102.0 \pm 10.4$ & 0.586 \\
\hline PR interval before TAVI [ms] & $165.8 \pm 25.3$ & $161.7 \pm 24.7$ & 0.516 \\
\hline PR interval after TAVI [ms] & $179.1 \pm 34.7$ & $173.0 \pm 20.1$ & 0.502 \\
\hline QT interval before TAVI [ms] & $419.0 \pm 34.2$ & $394.7 \pm 41.4$ & 0.062 \\
\hline QT interval after TAVI [ms] & $433.2 \pm 47.9$ & $448.8 \pm 45.0$ & 0.167 \\
\hline QTc interval before TAVI [ms] & $440.2 \pm 34.6$ & $423.2 \pm 30.1$ & 0.076 \\
\hline QTc interval after TAVI [ms] & $464.1 \pm 27.4$ & $445.7 \pm 35.8$ & 0.17 \\
\hline QRS axis before TAVI $\left[{ }^{\circ}\right]$ & $33.3 \pm 40.1$ & $3.8 \pm 31.4$ & 0.124 \\
\hline QRS axis after TAVI $\left[{ }^{\circ}\right]$ & $-5.5 \pm 47.1$ & $-27 \pm 22.3$ & 0.45 \\
\hline LBBB before TAVI, $n$ (\%) & $11(16.2)$ & $2(11.1)$ & 0.594 \\
\hline LBBB after TAVI, $n$ (\%) & $28(41.2)$ & $5(27.8)$ & 0.298 \\
\hline Patients with positive TPaVR before TAVI, $n$ (\%) & $14(20.6)$ & $6(33.3)$ & 0.255 \\
\hline Patients with positive TPaVR after TAVI, $n(\%)$ & $13(19.4)$ & $9(50)$ & 0.009 \\
\hline TPaVR before TAVI [mV] & $-0.7 \pm 1.7$ & $-0.5 \pm 1.5$ & 0.775 \\
\hline TPaVR after TAVI [mV] & $-0.5 \pm 2.1$ & $0.8 \pm 1.9$ & 0.039 \\
\hline STaVR before TAVI [mm] & $0.8 \pm 0.9$ & $1.1 \pm 1.1$ & 0.553 \\
\hline STaVR after TAVI [mm] & $1.0 \pm 0.6$ & $0.9 \pm 0.7$ & 0.48 \\
\hline TP/STaVR ratio* before TAVI, $n$ & $1.78 \pm 0.82$ & $2.84 \pm 2.29$ & 0.086 \\
\hline TP/STaVR ratio* after TAVI, $n$ & $2.21 \pm 1.52$ & $5.44 \pm 3.69$ & 0.002 \\
\hline
\end{tabular}

LBBB - left bundle branch block, TAVI - transcatheter aortic valve implantation, TPaVR - T wave polarity in lead aVR, STaVR - ST deviation in lead aVR. *TP/STaVR ratio: absolute value of bigger one (STaVR or TPaVR)/absolute value of smaller one (STaVR or TPaVR).

Table III. Comparison of patients' echocardiographic findings

\begin{tabular}{|c|c|c|c|}
\hline Parameter & Living $(n=68)$ & Deceased $(n=18)$ & $P$-value \\
\hline Pre-TAVI EF (\%) & $39.6 \pm 5.2$ & $41.1 \pm 4.8$ & 0.277 \\
\hline Post-TAVI EF (\%) & $43.4 \pm 8.9$ & $41.8 \pm 5.9$ & 0.483 \\
\hline $\operatorname{LaD}[\mathrm{mm}]$ & $43.3 \pm 3.7$ & $41.3 \pm 4.7$ & 0.443 \\
\hline Mean aortic gradient before TAVI [mm Hg] & $59.2 \pm 10.7$ & $56.1 \pm 10.1$ & 0.263 \\
\hline Mean aortic gradient after TAVI [mm Hg] & $12.1 \pm 2.8$ & $12.8 \pm 2.8$ & 0.381 \\
\hline $\operatorname{AVA}\left[\mathrm{cm}^{2}\right]$ & $0.8 \pm 0.1$ & $0.7 \pm 0.2$ & 0.279 \\
\hline Aortic annulus $[\mathrm{cm}]$ & $2.2 \pm 0.2$ & $2.1 \pm 0.8$ & 0.302 \\
\hline Sinus valsalva diameter $[\mathrm{cm}]$ & $3.1 \pm 0.2$ & $2.9 \pm 1.5$ & 0.21 \\
\hline Ascending aorta $[\mathrm{cm}]$ & $3.1 \pm 0.2$ & $3.2 \pm 0.6$ & 0.87 \\
\hline Paravalvular leak after TAVI, $n$ (\%) & $1(1.5)$ & $1(5.6)$ & 0.377 \\
\hline Prosthetic valve size [mm] & $25.6 \pm 2.5$ & $26.1 \pm 2.1$ & 0.401 \\
\hline Distance between aortic annulus and LMCA [mm] & $11.5 \pm 2.0$ & $8.2 \pm 1.1$ & $<0.001$ \\
\hline PAPs [mm Hg] & 44. $0 \pm 7.8$ & $40.0 \pm 13.2$ & 0.662 \\
\hline
\end{tabular}

AVA - aortic valve area, EF - ejection fraction, LaD - left atrial diameter, LMCA - left main coronary artery, PAPS - systolic pulmonary artery pressure, TAVI - transcatheter aortic valve implantation. 
Table IV. Comparisons of patients' angiographic findings

\begin{tabular}{|lccc|}
\hline Parameter & Living $(n=68)$ & Deceased $(n=18)$ & $P$-value \\
\hline LMCA, $n(\%)$ & $6(8.8)$ & $2(11.1)$ & 0.671 \\
\hline LAD, $n(\%)$ & $15(22.1)$ & $4(22.2)$ & 0.998 \\
\hline CX, $n(\%)$ & $13(19.1)$ & $3(16.7)$ & 0.812 \\
\hline RCA, $n(\%)$ & $10(14.7)$ & $2(11.1)$ & 0.696 \\
\hline SS, $n$ & $9.4 \pm 7.6$ & $7.2 \pm 7.4$ & 0.575 \\
\hline
\end{tabular}

CX - circumflex artery, LAD - left anterior descending artery, LMCA - left main coronary artery, RCA - right coronary artery, SS - SYNTAX score.

Table V. Independent predictors of mortality in TAVI patients

\begin{tabular}{|lccc|}
\hline Parameter & Odds ratio & 95\% confidence interval & $P$-value \\
\hline Calcium & 2.322 & $0.242-22.248$ & 0.465 \\
\hline Albumin & 3.994 & $0.236-67.633$ & 0.337 \\
\hline $\begin{array}{l}\text { Presence of positive TPaVR } \\
\text { Distance between aortic annulus and } \\
\text { LMCA (for each 1 mm increase) }\end{array}$ & 8.765 & 0.041 & $0.158-0.595$ \\
\hline $\begin{array}{l}\text { TP/STaVR ratio* after TAVI (for each } \\
\text { O.1 increase) }\end{array}$ & 1.966 & $1.276-3.024$ & 0.001 \\
\hline
\end{tabular}

LMCA - left main coronary artery, TAVI - transcatheter aortic valve implantation, TPaVR - T wave polarity in lead aVR. ${ }^{*} T P / S T a V R$ ratio: absolute value of bigger one (STAVR or TPaVR)/absolute value of smaller one (STaVR or TPaVR).

pro-BNP levels were significantly associated with 30-day and 1-year mortality in TAVI patients [21]. Some studies reported that paravalvular leak due to valve malapposition had negative effects on mortality and morbidity [22-24]. Both of our groups had similar STS-PROM scores. BUN and creatinine levels were similar in both groups, and there was no acute kidney damage in any of our patients. Both groups had higher pro-BNP levels than the normal population, but there was no significant difference between groups. EF measurements were also similar and less than $50 \%$ in both groups.

QRS enlargement, increase in PR distance, new onset $A F$, and complete atrioventricular block may occur in the TAVI procedure. It is reported that there was a high possibility of permanent pacemaker need in TAVI patients with post-procedural $\mathrm{QRS} \geq 120 \mathrm{~ms}[25,26]$. In a meta-analysis, it was reported that permanent pacemaker implantation incidence was about 15\% [27]. The QRS complex durations were increased in our patients when compared to pre-procedure ECGs, but there was no statistically significant difference in either group. Some studies reported that either pre- or post-procedural new onset AF had negative effects on mortality $[28,29]$. We did not include patients with AF and had no post-procedural newly developed AF. Patients with AF were excluded in case of possible deaths due to stroke. So, the AF effect on mortality was not investigated in our study.
The main limitation of our study was its retrospective design. Also, the small subject size is another major limitation. We did not perform serial hs-TnT measurements or control angiography in patients with subclinical myocardial damage. Calcium score was not calculated in any patient. Multislice computed tomography was not performed in most of the patients.

In conclusion, this study demonstrated the importance of surface ECG findings after TAVI. Patients with positive TPaVR, an increased TP/STaVR ratio after TAVI, and a short aortic annulus-sinus Valsalva distance should be closely monitored for mortality. There is a need for more extensive studies to identify the significance of this ratio in TAVI patients.

\section{Conflict of interest}

The authors declare no conflict of interest.

\section{References}

1. Osnabrugge RL, Mylotte D, Head SJ, et al. Aortic stenosis in the elderly: disease prevalence and number of candidates for transcatheter aortic valve replacement: a meta-analysis and modeling study. J Am Coll Cardiol 2013; 62: 1002-12.

2. Akin I, Kische S, Rehders T, et al. Indication for percutaneous aortic valve implantation. Arch Med Sci 2010; 6: 296-302.

3. Nishimura RA, Otto CM, Bonow RO, et al. 2017 AHA/ACC Focused Update of the 2014 AHA/ACC Guideline for the 
management of patients with valvular heart disease: a report of the American College of Cardiology/American Heart Association Task Force on Clinical Practice Guidelines. J Am Coll Cardiol 2017; 70: 252-89.

4. Lytvyn L, Guyatt GH, Manja V, et al. Patient values and preferences on transcatheter or surgical aortic-valve replacement therapy for aortic stenosis: a systematic review. BMJ Open 2016; 6: e014327.

5. Smith CR, Leon MB, Mack MJ, et al. Transcatheter versus surgical aortic-valve replacement in high-risk patients. N Engl J Med 2011; 364: 2187-98.

6. Leon MB, Smith CR, Mack MJ, et al. Transcatheter or surgical aortic-valve replacement in intermediate-risk patients. N Engl J Med 2016; 374: 1609-20.

7. Holmes DR Jr, Brennan JM, Rumsfeld JS, et al. Clinical outcomes at 1 year following transcatheter aortic valve replacement. JAMA 2015; 313: 1019-28.

8. Houthuizen P, Van Garsse LA, Poels TT, et al. Left bundle-branch block induced by transcatheter aortic valve implantation increases the risk of death. Circulation 2012; 126: 720-8.

9. Hoffmann R, Herpertz R, Lotfipour S, et al. Impact of a new conduction defect after transcatheter aortic valve implantation on left ventricular function. JACC Cardiovasc Interv 2012; 5: 1257-63.

10. Bleiziffer S, Ruge H, Hörer J, et al. Predictors for new-onset complete heart block after transcatheter aortic valve implantation. JACC Cardiovasc Interv 2010; 3: 524-30.

11. Kireyev D, Arkhipov MV, Zador ST, et al. Clinical utility of aVR-the neglected electrocardiographic lead. Ann Noninvasive Electrocardiol 2010; 15: 175-80.

12. Tanaka Y, Konno T, Tamura Y, et al. Impact of T wave amplitude in lead aVR on predicting cardiac events in ischemic and nonischemic cardiomyopathy patients with an implantable cardioverter defibrillator. Ann Noninvasive Electrocardiol 2017; 22: e12452. DOI: 10.1111/ anec. 12452

13. Al-Zaiti SS, Fallavollita JA, Canty JM, et al. The prognostic value of discordant $T$ waves in lead aVR: a simple risk marker of sudden cardiac arrest in ischemic cardiomyopathy. J Electrocardiol 2015; 48: 887-92.

14. İçen YK, Koç M. ST segment change and T wave amplitude ratio in lead aVR associated with coronary artery disease severity in patients with non-ST elevation myocardial infarction: a retrospective study. Medicine (Baltimore) 2017; 96: e9062.

15. Ducas R, Ariyarajah V, Philipp R, et al. The presence of ST-elevation in lead aVR predicts significant left main coronary artery stenosis in cardiogenic shock resulting from myocardial infarction: the Manitoba cardiogenic shock registry. Int J Cardiol 2013; 166: 465-8.

16. Yong ZY, Wiegerinck EM, Boerlage-van Dijk K, et al. Predictors and prognostic value of myocardial injury during transcatheter aortic valve implantation. Circ Cardiovasc Interv 2012; 5: 415-23.

17. Rodes-Cabau J, Gutierrez M, Bagur R, et al. Incidence, predictive factors, and prognostic value of myocardial injury following uncomplicated transcatheter aortic valve implantation. J Am Coll Cardiol 2011; 57: 1988-99.

18. Carrabba N, Valenti R, Migliorini A, et al. Prognostic value of myocardial injury following transcatheter aortic valve implantation. Am J Cardiol 2013; 111: 1475-81.

19. Stundl A, Schulte R, Lucht H, et al. Periprocedural myocardial injury depends on transcatheter heart valve type but does not predict mortality in patients after transcatheter aortic valve replacement. JACC CardiovasC Interv 2017; 10: 1550-60.
20. Czerwińska-Jelonkiewicz K, Witkowski A, Dąbrowski M, et al. Antithrombotic therapy - predictor of early and long-term bleeding complications after transcatheter aortic valve implantation. Arch Med Sci 2013; 9: 1062-70.

21. Giordana F, D'Ascenzo F, Nijhoff F, et al. Meta-analysis of predictors of all-cause mortality after transcatheter aortic valve implantation. Am J Cardiol 2014; 114: 1447-55.

22. Schewel D, Frerker C, Schewel J, et al. Clinical impact of paravalvular leaks on biomarkers and survival after transcatheter aortic valve implantation. Catheter Cardiovasc Interv 2015; 85: 502-14.

23. Takagi K, Latib A, Al-Lamee R, et al. Predictors of moderate-to-severe paravalvular aortic regurgitation immediately after CoreValve implantation and the impact of post-dilatation. Catheter Cardiovasc Interv 2011; 78: 432-43.

24. O'Sullivan KE, Gough A, Segurado R, et al. Is valve choice a significant determinant of paravalvular leak post-transcatheter aortic valve implantation? A systematic review and meta-analysis. Eur J Cardiothorac Surg 2014; 45: 826-33.

25. Akin I, Kische S, Paranskaya L, et al. Predictive factors for pacemaker requirement after transcatheter aortic valve implantation. BMC Cardiovasc Disord 2012; 12: 87.

26. Takahashi M, Mouillet G, Deballon R, et al. Impact of QRS duration on decision of early removal of pacing catheter after transcatheter aortic valve replacement with CoreValve Device. Am J Cardiol 2017; 120: 838-43.

27. Erkapic D, De Rosa S, Kelava A, et al. The risk for the permanent pacemaker after transcatheter aortic valve implantation: a comprehensive analysis of the literature. J Cardiovasc Electrophysiol 2012; 23: 391-7.

28. Tarantini G, Mojoli M, Windecker S, et al. Prevalence and impact of atrial fibrillation in patients with severe aortic stenosis undergoing transcatheter aortic valve replacement: an analysis from the SOURCE XT Prospective Multicenter Registry. JACC Cardiovasc Interv 2016; 9: 937-46.

29. Amat-Santos IJ, Rodés-Cabau J, Urena M, et al. Incidence, predictive factors, and prognostic value of new-onset atrial fibrillation following transcatheter aortic valve implantation. J Am Coll Cardiol 2012; 59: 178-88. 